\title{
MULTIPLE NEURITIS, ILLUSTRATED BY CLINICAL CASES.
}

\author{
By J. MAGEE FINNY, M.D. Dubl.; \\ President of the Royal College of Physicians of Ireland ; \\ Clinical Physician to Sir Patrick Dun's Hospital.
}

[Read in the Section of Medicine, November 21, 1890.]

THE subject which I wish to bring under the notice of the Academy is one of very considerable interest, and one not altogether devoid of novelty. It is, moreover, one which is not as well known as it deserves to be, and in connection with it every fresh case recorded will probably enlarge and render more accurate our information on some point or points in its ætiology or pathology.

I must, at the same time, crave your indulgence if the remarks I may make, and the conclusions I deduce, have been already and better made, in their writings and communications, by those who may be looked upon as authorities on such subjects, in the literature of the subject in the journals and works on nervous diseases.

It may be well, for the better elucidation of the subjectthough quite unnecessary-to remind you that paralysis of motion or of sensation, or of both, may be due to either central disease of the cerebro-spinal axis, or to injury of or disease of the peripheral nerves. Thus, a hæmorrhage into the brain substance, or the motor paths in the pons medulla or spinal cord, will manifest its presence by paralysis of motion of the muscles of the limb or limbs supplied by nerve force from these nerve centres; and again, the muscles of a limb may be powerless from injury or inflammation of the nerve trunks as they pass out from the cord, or of the nerves themselves, as they directly end in the muscles. In either case the limb is paralysed, but the seat of the disease and its nature are widely different. Hence the division of paralysis, as 
to whether it be "central" or "peripheral" in its seat, is both pathologically correct and clinically useful.

Extended clinical observation bas met with many cases of paralysis of the extremities which could not be attributed to transverse myelitis, to the spinal degenerations of locomotor ataxy or spastic paraplegia, or to the rarer hæmorrhages into the spine, the presence of new growths, or the results of traumatism.

The cases to which I refer differ in their symptomatology and their clinical history and course so widely from those cases due to central disease that until the existence, as an entity, of peripheral nerve inflammation came to be recognised, very great difficulty was felt by clinical observers in classifying the paralyses met with in practice. Indeed, every physician who is in charge of a hospital, and enjoys a moderately wide practice, cannot but occasionally meet with cases of paralysis, involving motion and sensation to a greater or less degree, which he has considerable difficulty in recognising and greater difficulty in referring to any of the generally accepted pathological conditions of the brain and spinal cord. These difficulties I have myself experienced on many occasions, and in bringing under the notice of the Academy a few illustrative cases, which occurred within the last year, I feel sure I shall have the sympathy of some whose experience has been similar to my own; and I trust I shall elicit from others, whose special training in nervous diseases is wider and more cultured than I can lay claim to, much helpful criticism and useful information.

The cases of paralysis to which I particularly refer have certain general features of resemblance which connect them together in one group, and which help to distinguish them from paretic conditions, the result of central disease in the spinal cord or the brain. The most prominent of these symptoms is pain in the nerves and in their distribution to the muscles and the skin; and it is this early pain and paralysis, and its symmetrical distribution in the extremities, which gives the title of multiple neuritis, or, as the result of inflammation of the nerve is that the paralysed muscles atrophy and degenerate, it is sometimes spoken of as multiple degenerative neuritis. 
As a rule, the disease shows itself first in the feet and legs, and then in the hands and forearms, and its course and duration is chronic or subacute, lasting months and even years. Its termination also is usually in more or less complete recovery. This chronic course is, however, not invariably the case, as occasionally, and fortunately most rarely, the onset is sudden, paralysis attacks the feet and legs, and, rapidly involving the hands and arms, attacks the trunk and muscles of respiration, and ends fatally by suffocation and exhaustion within a few days, or a week at furthest. To this remarkable disease is given the name of acute ascending paralysis, first described by Landry.

Some years ago one such case came under my care in the City of Dublin Hospital. It was in the person of a young woman, aged twenty-five; and it ran its course in the manner $I$ have described in about six or eight days.

I had never seen such a case before, nor have I met with one since; but from what $I$ knew by study $I$ took it to be one of acute ascending paralysis of Landry, and as such $I$ published it in the Brit. Medical Journal in 1880.

A macroscopic examination of the spinal cord showed nothing abnormal, and I simply recorded it as an example of a strange and little-known disease. Since then and within the last twelve months, however, the pathology of Landry's paralysis has been the subject of many communications both at home and abroad, and various views as to its nature and pathology have been promulgated. Dr. Ross has most fully discussed this question in the pages of the Medical Chronicle ; ${ }^{2}$ and, basing his opinion upon the records of ninety cases which he has collected and analysed, he has come to the conclusion that the disease is not one of central origin-not an ascending central myelitis-but one of peripheral neuritis. Strümpell states that many cases of Landry's disease are really cases of multiple degenerative neuritis, while Nauwerck and Barth $\mathbf{b}$ go further, and say-" It has not been proved, even in the extended

- Medical Chronicle. Vol. XI. Nov. 1, 1889.

- Quoted by Ross. Ibid. 
signification of the term, that acute ascending paralysis can be caused by disease of the central nervous system."

It is, however, not of "acute multiple neuritis" that $I$ intend to speak (though, doubtless, much interest attaches to it), nor of the varied manifestations of neuritis known by the name of kakkè of Japan, or the beriberi endemic to many tropical or subtropical places; nor will I refer to leprous neuritis, nor to those paralytic conditions which have been met with in and following upon diphtheria, advanced cases of typhus and typhoid fevers, and the terminal stages of pernicious anæmia.

I wish rather to bring under the notice of the Academy some examples of the chronic or subacute disease which $I$ have referred to as that which attacks the nerves of the extremities-either the feet and legs, or the hands and forearms, or both the upper and lower extremities together, with a remarkable symmetry, and of which the involvement of the sensory, vasomotor, and secretory nerves form a most prominent and distinguishing feature.

The ætiology of the disease is undoubtedly obscure, and in many instances quite unknown. One of my cases can be directly referred to exposure to cold, and in another subacute articular rheumatism seemed to play a part; while, again, a third was directly due to the poison of alcohol, which is acknowledged to be the most common cause of the disease, especially in women; and yet another may owe its origin to the obscure and nerve-depressing influence of Russian influenza. It seems to be a disease of adult life between thirty and fifty years of age, and with the exception of one case of a young man, aged twenty-three, my cases conform to the rule.

The pathological condition of the nerves and nerve centres is as follows (I quote from "Gowers' Manual of Diseases of the Nervous System," 1886, Vol. I., pp. 95, 96) :-“"The distribution of the nerve changes, which are chiefly degenerative, varies in different cases; but, as in simple neuritis, the nerve inflammation is best seen at certain spots-where, for instance, a nerve pierces the fascia or winds round a bone. The nerves of the limbs only suffer in the majority of cases. The corresponding nerves on the two 
sides are usually affected. The musculo-spiral nerve in the arm and the anterior tibial nerve in the leg usually suffer first and in greatest degree, but often all the nerves of the limbs are involved. The spinal cord has been found healthy in most cases, though now and then more considerable changes (usually those of chronic myelitis) have been found in the cord. They are probably merely coincident."

From the foregoing brief summary of its pathology it will be readily seen that the prominent symptoms should be those of motor paralysis and sensory irritation; while from the special involvement of the musculo-spiral nerve and the anterior tibial nerve the paralysis will be chiefly of the extensor muscles of the hands and feet, producing drop-wrist and drooping of the foot, more especially of its outer side.

I shall now relate to you the cases I have met, and thus practically illustrate the particular features of the disease :-

CASE I.-Multiple Neuritis affecting the Hands and Arms, with perversions of sensation, and vasomotor and secretory disturbances.James P., aged fifty-five, storekeeper, was admitted under me into Sir Patrick Dun's Hospital on July 21, 1890.

Twelve months ago he experienced a numbness in the second finger of his right hand, and soon afterwards in the other fingers of that hand, and then in the fingers of the left hand also.

About the same time a stinging "leaping" pain was felt in the distal palmar surfaces of the fingers, and for weeks at a time hard black spots appeared on the finger ends (the thumb alone being exempt). Considerable tenderness and pain on closing the hand was experienced, as well as shooting neuralgic pains at times through the fingers thus affected. These pains did not synchronise with the numbness, but sometimes the pain and sometimes the numbness predominated. He was, however, able to work as a fireman in charge of a small engine. He complained at that time of the effect of cold in his hands, and that on a cold night his fingers became deadly cold and white in colour.

Five weeks ago pains, with a feeling of trembling, attacked his forearms and arms as high as the shoulder, and off and on they still occur, being principally referred to the deltoids. The pain does not seem to follow any particular nerve, nor does deep pressure 
on the muscles produce pain. When the pain is severe in the arms, deltoids, and shoulders, the patient thinks his hands become blue and colder. The coldness and blueness of the hands and fingers is very marked, and more pronounced when the patient is up and about than when he is in bed, but even while in bed in the month of June this change was very perceptible. The skin over the fingers, front and back, is glossy and tight, so that it cannot be moved or pinched up from the underlying tissue.

Some days after admission to hospital, and the application of massage, the hands and fingers became bathed in perspiration. They no longer trembled, and were warmer than they had been for months. The grasp of the hand was fairly good, though less than might be expected of a labouring man, but the finer movements of the fingers were imperfectly performed, and a decided feebleness of the forearms and arms was present. On exposure of the deltoid vermicular muscular movements were visible. There was no anæsthesia discoverable anywhere, to touch or temperature, or to pricking with a pin.

Unfortunately the hospital batteries were not in order, so that the electrical reactions could not be tested.

Full inquiry into the history or antecedents of the patient failed to elicit any probable cause of the disease, as there had been no previous illness or sore throat, nor was he exposed to any toxic influences, such as lead, and he denied having taken much drink, or having had syphilis, and careful examination of the organs and viscera showed he was free from organic disease.

The patient remained under observation a little over a fortnight, but being dissatisfied with the progress towards recovery he left very much in the same state as when he was admitted to hospital.

The symmetry of the disease, the shooting pains in the fingers and arms, the cold blue fingers and hands with profuse perspiration, the altered nutrition of the skin-all pointed to neuritis affecting the sensory as well as the motor nerves of the forearms and hands; and the case may be considered as an early stage of the disease, or, perhaps more correctly, a modified form of the disease with a limited area of distribution.

The next case I would refer to is a much more aggravated one, in which the symptoms of pain, tenderness of the limbs, and increased sensitiveness of the skin were most pronounced. It differed in some respects, and to a marked degree, from the usual 
features of multiple neuritis, and I am quite aware that the presence of the tendon reflexes, faradic excitability of the muscles, and the very moderate muscular paralysis of the extensors of the wrists and ankles, give full grounds for questioning the propriety of including it in the class of nervous diseases to which $I$ was led to refer it.

I am, however, quite at a loss to know under what other pathological conditions it could find a place, since the history of its onset, its distribution, course and progress to recovery, removed it from all central diseases of brain or spinal cord. Moreover, with special reference to the myotatic irritability which was present, it is not at all improbable that it may have been superadded upon pre-existing degenerative changes in the spinal cord, with some chronic meningitis. Be that as it may, I find that Dr. Thomas Buzzard ${ }^{a}$ states that it will now and then happen that the kneereflex may be not only present but somewhat exaggerated, and he records a case of alcoholic neuritis in furtherance of the view, and quotes another related by Dr. Oettinger, in which the knee phenomenon is described as "notably exaggerated." In the sequel of this case it is right to add that the knee phenomenon disappeared.

CASE II.-T. M'L., aged twenty-six, a labourer, from county Mayo, was admitted to Sir Patrick Dun's Hospital on June 20th, 1889 , complaining of weakness of the extreinities, with soreness and tenderness of the hands and feet.

'Two years ago he was first attacked by a stabbing pain in his left hip, which he attributed to a chill he got while sleeping in a cold room. The pain remained in the hip for about a fortnight, and then ran down to the foot. He was treated for sciatica and had the hip blistered. Since then the pains have been alternately in his feet and in his hands-in the palmar surface of the latter and the dorsum of the former. Three months ago the pains and tenderness had greatly increased in his hands and feet, and his powers of using them decreased, so that now he cannot stand on his feet or open his fingers to grasp with his bands, or use a stick to support himself, or even employ them for raising himself in his bed. Hence his method of lifting himself to sit up in bed is

- Paralysis from Peripheral Neuritis. 1886. Pp. 8586. 
peculiar, as he half rolls on his face and uses the under-elbow to act as a lever for that purpose. The left hand and forearm are less affected than the right. The patient says there are certain "blue spots" which are specially painful, and that these vary at times, and to these he attributes all the trouble.

Examination partially confirms this, as there are certain slightly reddish patches, with blue veins running through them, seen on the joints, such as the wrists and ankle-joints, as well as on the ball of the thumb and the sole of the foot. The colour of these spots was seen to vary under observation, and certainly when they faded or disappeared less pain was present. They also changed their position, and I failed to explain their occurrence by any theory of cutaneous distribution of the nerves or of blood-vessels, and they were quite unlike any gouty or rheumatic affection, or neuromatous nodules. The hands and feet were frequently bathed in perspiration, and when wiped dry were seen in a few seconds to be again covered with beads of moisture. This symptom also varied in its intensity. The fingers were kept constantly flexed in the palm, the thumbs being similarly, though less, affected, and the patient had no power to extend them, and if the fingers be forcibly opened he suffered great pain. In like manner the toes were flexed and the ankles extended, and any forcible flexion of the latter or extension of the former gave pain and caused outcry. The skin over the feet and fingers was very smooth and glossy, and could not be readily pinched up or moved. The muscles of both the forearms and the legs were atrophied, but owing to the extreme tenderness and pain their individual power could not be satisfactorily tested. Sensation seemed unaffected as to touch, to pain of pricking with a pin, and to temperature. The tendon reflexes were present, and on the left side seemed to be rather exaggerated ; while ankle clonus was elicited in the right leg. The patient had full command over the bladder and rectum, and there were not any bedsores. The urine was healthy - free from albumin and sugar ; and there was an absence of all defects of hearing, sight, or speech, and except for an exaggerated interest in his symptoms, and attributing all his ailments to the "blue spots" already referred to, his mental and psychical functions seemed normal.

There is no history or evidence of either his having had syphilis or of being given to alcoholic excess.

The further progress of the case was towards recovery. The treatment adopted consisted of potassium iodide in combination 
with small doses of mercuric chloride, and daily massage to all the extremities. The left hand was improving fast when the pain returned owing to the patient having hurt it while dressing. In four weeks the patient was able to bear his weight on his feet, and by the aid of sticks to move about for two or three yards at a time; and by the middle of September he was able to dress himself, to get out of bed, and to walk without much pain about the ward. The pains varied, and after walking rather too much they returned for some days in the feet. The left hand he was able to open quite freely, and to grasp with it fairly well, but the fingers of the right remained flexed. The excessive perspirations ceased and the painful spots disappeared. Faradic electricity was applied to the muscles of the forearms in August by my colleague, Dr. Smith, under whose care he was for a time, with the result that muscular excitability of both arms was almost identical, but the force of contraction was considerably less in the left arm. It is right to mention also that ankle clonus was elicited about the same time in the right leg, but this was not a constant symptom, nor could it be looked for until the pains of moving the ankle had disappeared.

The patient left hospital on October 18th, after about four months' sojourn and treatment, very much improved in all respects.

CASE III.-W. K., aged twenty-three, farmer, was admitted from the country under me into Sir Patrick Dun's Hospital on September 4th, 1889, suffering from complete loss of power in both legs, with pains in his shins and knees.

The patient is a tall, fair-haired man, six feet high, who, if his hands and legs were not seen, might readily pass for a healthy individual. He was unable to stand or walk without support, and when be attempted to walk it was noticed that he lifted the knee very high, and that the outside of the foot and toes had apparently a tendency to cling to the ground, and they struck the ground first on the foot being brought down again, and the great toe doubled under the sole. There was no ataxia. The patient cannot attribute the onset of the disease to any particular cause, except it be to wet and cold. He states that he experienced pains in his bead last October. Leaving the head, they went all over his chest and back, so that by December, 1888, he had pains all over him. Then for three months be experienced no loss of power of movement, but in March of the year 1889 the pain became continuous in his feet and legs, and he completely last the power of movement of his 
limbs. He describes the pains of his legs as running down the shin bones and round the knees symmetrically, those in the right leg having preceded those in the left leg by some few days. Simultaneously with the appearance of the pains in his legs, darting pains shot round to his back, but there was no description of "girdle pains." The legs when examined were found greatly atrophied and particularly along the tibial aspect, the extensor muscles in the thigh being also much wasted. The feet hung down like the "dropwrist" of lead palsy, the toes being flexed and the foot inverted. The great toe projected above the others, and seemed to have been injured while attempting to stand or walk, by striking against some obstacle. Indeed, while in hospital, he produced nasty bleeding sores at the root of the nail of the great toe of either foot by striking it against either the floor or his bed while attempting to get out of bed or walk to the fire. The atrophied muscles gave no response to a very strong faradic current, and galvanism in like manner gave negative results when applied to the muscles of the legs. The forearms were also feeble and wasted, but to a much less degree than the legs, and responded better to the galvanic than to the faradic stimulus. The excitability of the nerves remained while that of the muscles was lost. With the exception of the outer side of the left foot, sensation was completely lost in the legs below the level of the patella on the inside and a little lower down on the outside. There is an excessive growth of hair on the legs, particularly about the knees, and the feet are constantly bathed in clammy perspiration. Thus at the same time anæsthesia coexists with neuralgic pains and tenderness along the shin bones. The knee reflexes were quite abolished. The hands and forearms are much less affected, but the wrists and fingers "droop" considerably, and the patient finds difficulty in performing such actions as using a pin, \&c. The grasp of the hands, as tested by the dynamometer, is very defective. There was no want of power over either the bladder or the rectum, nor were there any bedsores.

The treatment consisted of massage and electricity-both galvanism and faradaism-every day, and distinct improvement was noticeable shortly afterwards. He was also given iod. potass. with perchloride of mercury.

The urine, which was generally acid, was found, on September 13 , to be faintly alkaline, though free from either albumin or sugar. This alkalinity was, however, transient.

On September 25 he was able to be up each day, and by the aid. 
of a friendly arm was able to take a few steps, and he made slight improvement in his powers of locomotion ; and in November, when he went home to the country, his feet had ceased to sweat, also the nerve pains of the knees and shins were considerably less, and were the first symptoms to yield to treatment.

I heard from this patient in February, 1890, and he reported some, but not much, improvement. Again in May he wrote to say he could stand without extraneous help ; and yesterday (Nov.9) I heard from him the following more accurate details, in reply to some queries I put to him, which show decided, though gradual, progress towards recovery. His hands and arms are, he says, quite strong, and his legs are improved, so that he can stand alone. The sweating of the hands and feet has ceased, with the exception of the toes of the left foot. The feeling in his legs and feet is not yet natural, and he has still some of the pains, but to a much less degree, and the sores on the great toes are not healed yet. He has no trouble in making water, though at times it is slow in passing.

The question of diagnosis in this case lay probably between multiple neuritis and spinal leptomeningitis. The preceding pains of some months' duration, followed by motor paralysis, atrophy, and the loss of sensation, might be referable to either causation; but the symmetrical neuralgic pains of the knees and tibiæ, the absence of all spasm or muscular contractions, the "drop" feet, the perspirations of the feet, the glossiness of the skin of the digits and the dorsum of the feet, along with the loss of patellar reflexes and the presence of complete control over the bladder and the rectum, formed a group of symptoms which belong to multiple neuritis, and which could not be produced by any central lesion, so far as I know.

The next case is an example of alcoholic multiple neuritis:-

CASE IV.-A lady, aged fifty, with several children, was seen by me, in consultation with Dr. S. T. Gordon, twelve months ago.

The history was unmistakably one of alcoholic excess of some years' duration, as much as a gallon of whisky having been consumed in three or four days. Dr. Gordon first attended her three weeks before she came under my observation, and he saw at once the character of his patient, and that she had become so addicted 
to drink that all the methods of bribery of servants, falsehoods, and deceptions, were being employed to obtain it.

Dr. Gordon had, however, restricted the amount to two glasses a day by means of a reliable nurse.

Her symptoms were, at first, seemingly those of alcoholism-as vomiting, a dry red tongue, with hepatic tenderness and enlargement, were the marked symptoms. Her manner and speech was peculiar, so as to suggest hysteria, while her temper was most variable, and at times so fitful and violent as to point to dementia.

Five weeks ago she complained of pains in her fingers, wrists, ankles, and toes, especially the big toe, and these were looked upon as being due to rheumatic gout-a view which gained confirmation by the history of her having had rheumatism five years ago, and the presence of a heavy cloud of lithates in the urine.

The symptoms and features and progress of the case, when seen by me, and subsequently to my visit, tally so closely with those of alcoholic multiple neuritis, that I shall simply refer to them in the order of their prominence and sequence.

At the time of my visit this lady was confined to bed, being unable to get out of bed or to stand. She seemed also quite unable to help herself with her hands, which she moved about in an awkward and almost affected manner. It was seen that the hands dropped at the wrists (somewhat like the drop-wrist of lead palsy). The extensor muscles were almost completely paralysed, and when she attempted to grasp with the hands she complained very much of the pain in her wrists and forearms. Movement of the legs also gave her pain, and caused considerable outcry, the pain being especially referred to the front of the tibix and across the toes, and particularly to the balls of the great toes. The muscles of both legs and forearms were wasted, the extensor aspects respectively showing the degenerative changes, and the motor power was decidedly deficient. Pressure over exposed nerves, such as the median and the peroneal, was extremely painful, while the muscles were sore to handling and deep pressure. Areas of diminished and again of exalted sensation were detected in the legs.

"Cutaneous hyperæsthesia is sometimes met in peripheral neuritis," says Dr. Ross,a "but it is quite overshadowed by an intense hyperæsthesia of the muscles, so much in some cases as to evoke loud cries from the patient on the slightest pressure."

The knee reflexes were completely absent. On account of the

Ibid. P. 178. 
tenderness and soreness of the feet no examination could be made as to the presence of ankle clonus.

The pulse was always quick, 120-140. There was complete absence of fever. The tongue was red and "irritable," and the bowels were irregular, though there was no paralysis of the sphincter ani. There seemed to be, however, paralysis of the bladder, as she passed her water involuntarily under her. She was subject to various delusions, I was informed, and her manner during my visit was peculiar-such as one might expect in a person who had taken too much wine-half-maudlin, familiar, and semi-hysterical. This manner, however, was not due to excess, as Dr. Gordon had restricted the quantity to moderate bounds, and a careful and reliable nurse carried out his instructions. A similar peculiarity of manner $I$ noticed in the case of acute ascending paralysis of Landry, to which I have alluded.

The subsequent course of this case Dr. Gordon has kindly furnished me with, and it is gratifying to know that it ended in recovery.

The first sign of improvement was a cessation of the delusions; then she regained power over the bladder. The pains in the legs and hands next became less severe, though they have completely left her only last month (October). Even after these pains diminished, the power of standing had not returned, and she had to be carried in a chair down stairs; and the power of writing was so defective that it was with the greatest difficulty and ingenuity that she was enabled to affix her signature to an important document requiring it. In September she was well enough to go to Buxton for a month, although she still suffered from pain in both tibiæ, but in the left particularly, and walked with the greatest difficulty. She returned to Dublin at the beginning of this month, greatly improved, and is now able to walk into town, a distance of over a mile, and to go shopping. She still has pains across the toes, and has to wear large soft boots; otherwise she is in excellent health.

It is right to state that on two occasions, at least, the progress was arrested and retarded by her having managed to elude the watchfulness of those about her, and to get whisky, but when again under restraint she rapidly regained the ground she had lost.

The treatment adopted in this case was principally the withholding of alcohol, the use of anodyne liniments to the painful parts, the internal administration of iodide of potassium and the 
perchloride of mercury, and the employment of massage and faradic electricity. To these latter Dr. Gordon attributes most credit.

Few words of comment are necessary to add to the description of this case. I wish, however, to call attention to one symptom unusual to general multiple neuritis and to the alcoholic formnamely, the loss of control over the function of the bladder, inasmuch as it has been generally accepted that the absence of this symptom is looked upon as a distinguishing feature of peripheral from central paralysis.

One may suggest possibly coincident central disease, or some form of reflex paralysis, or another phase of sensory perversion of the meatus as explanations, but at best they are but theories, and I prefer to simply record the fact and wait for further light to say whether this symptom of incontinence of urine may not be found as an occasional, though recognised, symptom of multiple neuritis.

I cannot pass from this case without noting its favourable termination, and the all-importance of removing the poison which seemed to produce it. The prognosis in every case of multiple neuritis may in general terms be stated as favourable to its ultimate issue, but each case must be measured by the limits of its causation, the amount of paralysis, and the general health of the patient. Some cases may recover rapidly, others more slowlyperhaps extending over three or four years. Some may recover completely, or again others incompletely, leaving deformity from contractions of muscles.

It has been frequently noticed that pulmonary phthisis has been a concomitant, and of ten the cause of a fatal issue in multiple neuritis, and this coincidence, if not relationship, should not be lost sight of in dealing with the subject of prognosis.

The last case I would bring under your notice is a sad example :-

CASE V.-A. B., aged twenty-eight, the mother of five children, the youngest eight months old, was seen by me in consultation last June (1890), and appeared to be in the last stage of multiple neuritis, which had ascended to the intercostal and facial muscles, and seemed to threaten death by asphyxia. 
When I entered the sittingroom in which this lady lay on her back, sunk down on a sofa, with her feet extended beyond it, I was struck by the drop-wrists, as her arms lay semiflexed across her chest, and by the downward and inward position of the feet, so that the plantar surfaces looked towards each other. I had little difficulty in arriving at the conclusion that the lady was the subject of multiple neuritis.

A more careful examination confirmed this hasty opinion.

There was no general paralysis, as she could flex the forearms, and her grasp was fairly strong. In like manner she could flex and extend the thighs, but the feet and toes were absolutely powerless, and she was quite unable to stand or move them; and there was also marked wasting of the muscles of the legs, especially of the extensors; those of the forearms were but slightly atrophied, and this was explained by the fact that the wrist-drop had only appeared a few days ago. The reflexes both superficial and deep were absent, and there was no ankle clonus. Sensation was very deficient in the feet, and on the dorsum of the second and third toes of the right foot a vesiculo-pustular rash existed. The bladder and rectum were under control, although cccasionally she wet herself. Flexing the foot gave rise to much pain, and deep pressure over the muscles of extension of the foot and toes as well as along the course of the musculo-spinal nerves in the arms was very distressing.

The patient spoke in a thick and indistinct manner, and during the day I learned she had had many delusions and had spoken to imaginary persons. A short purposeless cough occurred every few minutes, showing very little expulsive power of the thorax, and the act of inspiration was feeble and of expiration sudden and jerky. Her pulse was very shabby-130 in the minute, and the heart-sounds feeble; temp., 101\%. Physical examination of the lungs failed to show any active disease, though percussion in the left subclavicular region was comparatively less resonant.

The view I took of this lady's case was that it was an aggravated one of multiple neuritis, and, in the absence of diphtheria as a cause, I considered it probably alcoholic in its origin. I advised abundance of liquid nutriment, including 4 ozs. of wine, bark and ammonia, with potass. iod., and the employment of massage to the whole body.

As to the probable ætiological factor, the medical man, her physician, concurred in the view I ventured to suggest, as for some 
time he had suspected that she had been in the habit of taking, or of being supplied with, port wine in large quantities whenever she seemed weak or nervous, and that probably four to six glasses was the daily average for some weeks.

It is, however, quite possible that the lesion may have been the direct outcome of the influenza from which she suffered in January, and after which she never felt strong.

This gentleman has most kindly supplied me with a complete history of this sad case, from its active onset in April to its termination by suffocation in September, and I cannot do better than briefly summarise his information and notes of the case, inasmuch as there are divergencies in some symptoms from those peculiar to multiple neuritis, and some additional features are superadded.

The lady had an attack of influenza in January, which left after it considerable weakness, and her nervous system was still further depressed by the sudden death of her father some short time before. Her symptoms and manner were, however, very like those of hysteria, and to this condition were attributed the pains in her legs, with some hyperæsthesia of the skin, of which she complained in April. Soon after she spoke of numbness in her feet her memory became defective, and she suffered from double vision and inability to read or sew.

On April 28th her condition became decidedly worse, as she fell on one occasion in coming down stairs, and she could hardly stand alone or walk. Her speech also was noticed to be thick. She could converse intelligently, but would forget almost immediately what she had been told. Pains of a paroxysmal shooting character now set in. Commencing in the lower limbs, they shot up the thighs and across the abdomen.

In the middle of May, in addition to the foregoing symptoms, which became worse, deep pressure on the muscles gave pain; at the same time blunting of tactile sensation was present in the feet and legs. She says she could move her legs but for the agony it caused her to do so. The pains were more severe at night and prevented her sleeping.

In the beginning of June strict orders-though it was doubtful if they were carried out-were given to stop the free allowance of port wine, of which her medical attendant was then for the first time made aware.

I saw her on June 27th, and by the aid of a skilled and trustworthy nurse the stimulant was reduced to one glass of port wine 
in the day and half a glass of whisky in egg-flip, and in a fortnight afterwards was completely stopped.

In July the forearms began to regain some power, as she could slightly extend the fingers, and there was no pain, although wasting of the extensor muscles of both forearms and legs was manifest.

In the month of August no change was noted, except that the pains in the legs had disappeared, and that the general condition was decidedly worse.

On the 2nd and 5th of September suffocative attacks set in, as her thoracic muscles of respiration were completely paralysed, and movements almost $n i l$, and in these she nearly died. However, she rallied out of them, and although she passed her water under both before and during them, she was able to use the pan when directed to do so. Fæces, however, passed unconsciously.

On September 8th she became very restless and delirious, being under delusions as to the presence of persons in the room, with whom she held conversations. She was suspicious, and looking under the bed-clothes, and from side to side. She tossed her head about, sang hymns, and, in short, was a maniac. For forty-eight hours this restlessness persisted, and after two attacks of semicoma, in which Cheyne-Stokes' respiration was well marked, she died quietly on September 11th.

Examinations of the lungs and sputa gave negative results as to the co-existence of tubercle, and the urine was free from albumin, though frequently tested.

Everything that could be thought of was tried in succession to bring down the pulse and temperature, but completely failed to have any such effect. An inspection of the temperature charts shows that a fever, ranging from $101.5^{\circ}$ E. to $98^{\circ}$ M., ran on uninterruptedly from June 27 th to the termination.

The foregoing cases are, $I$ consider, fairly representative of the disease under consideration, and of the various types I have myself met with. I trust, in the discussion to follow, some members will be able to add much more to our information on this subject, and thereby supply the deficiencies of my communication, and awake the interest which my paper lacked, and of which no one is more conscious than I am myself. I thank you much for your patient hearing and indulgent attention. 
Dr. S. T. Gondon said the lady whose case the President had described, and who had been a patient of his, was now almost perfectly recovered. He had been visiting her a considerable time before he elicited that she was addicted to taking large quantities of alcohol. On examination she exhibited the typical drop-wrist, while her feet could not bear any weight, and she was also subject to delusions-for instance, she would repeat imaginary conversations. She had pains first in both tibiæ, then in her feet, particularly in the great toes, and then in the wrists and hands, while for some time she involuntarily passed urine. Under treatment the pains left her in the same order, but having again got some alcohol surreptitiously the pains returned, attacking her feet across the toes. Nine months had elapsed since the President saw her, and in the interval she had been for some time at Buxton, and had given up alcohol. Her mind was now quite clear, and though she still complained of pains, she went to a dance recently, and speaking from his knowledge of her for a number of years, he had never known her in so good health, mentally and physically, as she enjoyed at present. 